Original Paper http://ajol.info/index.php/ijbcs http://indexmedicus.afro.who.int

\title{
Chorologie des Stomoxyinae (Diptera) dans le Parc National de Moukalaba Doudou (sud-ouest Gabon)
}

\author{
Ely S. DIBAKOU ${ }^{1 *}$, Franck MOUNIOKO ${ }^{1}$, Christophe R. ZINGA-KOUMBA ${ }^{2,3}$, \\ Ornella A. MBANG-NGUEMA ${ }^{1}$, Geneviève ACAPOVI-YAO ${ }^{4}$, Bertrand M'BATCHI ${ }^{1}$ et \\ Jacques F. MAVOUNGOU ${ }^{1,2}$
}

\author{
${ }^{1}$ Université des Sciences et Techniques de MASUKU, BP 941, Franceville, Gabon. \\ ${ }^{2}$ Institut de Recherche en Ecologie Tropicale (IRET), BP 13354, Libreville, Gabon. \\ ${ }^{3}$ Ecole Régionale Post-Universitaire d'Aménagement et de gestion Intégrés des Forêts et Territoires Tropicaux \\ (ERAIFT), BP 15373, Kinshasa, R. D. Congo. \\ ${ }^{4}$ Université Félix Houphouët - Boigny, UFR Biosciences 22, BP : 582 ; Abidjan 22, Côte d'Ivoire. \\ *Auteur correspondant ; E-mail : sergeely@yahoo.fr
}

\section{RESUME}

Bien que l'importance des glossines dans la transmission des trypanosomes soit connue au Gabon, elle a longtemps masqué celle des stomoxes. C'est dans ce cadre qu'une étude sur la chorologie des Stomoxyinae a été conduite, en grande saison sèche et à l'aide de pièges Vavoua, dans un ancien foyer de la Trypanosomose Humaine Africaine (THA) en particulier dans le Parc National de Moukalaba Doudou (PNMD). Au total 14 pièges Vavoua ont été placés dont 7 au village Doussala et 7 en forêt que sont les deux biotopes prospectés dans le PNMD. L'enquête entomologique a permis de mettre en évidence six (6) espèces de stomoxes qui vivent en sympatrie avec des densités apparentes (DAP) différentes suivant les espèces. En effet, Stomoxys omega avec une densité appaente par piège de 47,94 a été l'espèce la plus abondante, suivi de Stomoxys niger niger $(\mathrm{DAP}=19,49)$. Les autres espèces à savoir Stomoxys xanthomelas, Stomoxys niger bilineatus, Stomoxys calcitrans et Stomoxys transvittatus ont été très faiblement représentées avec des densités apparentes respectives de 0,$25 ; 0,23 ; 0,012$ et 0,004 . La forte abondance des stomoxes dans ce parc nous interpelle à considérer ces insectes dans la lutte contre la THA dans cet ancien foyer.

(C) 2015 International Formulae Group. All rights reserved.

Mots clés : Stomoxes, Vavoua, Doussala, Parc, Gabon.

\section{INTRODUCTION}

La zone équatoriale d'Afrique centrale ou «bassin du Congo », abrite la plus grande forêt tropicale humide du monde après l'Amazonie (Bergonzini et Lanly, 2000, PFBC, 2006; IRD, 2011). La diversité des conditions écologiques ainsi que les singularités de son histoire biogéographique lui confèrent une importance stratégique pour la préservation de la biodiversité africaine et mondiale (FAO, 2002 ; PFBC, 2006 ; Akoma, 2014). Aussi, le Gabon pour répondre à cet 
enjeu majeur, a mis en place treize (13) parcs nationaux (Vande, 2006, 2012). Plus de 10\% de la superficie du pays leur est consacré afin de préserver la biodiversité et promouvoir l'écotourisme. Dans ces parcs, les animaux sont protégés et par voie de conséquence la taille des populations animales va augmenter et les contacts entre populations humaines et animales seront facilités. De plus, certains de ces parcs, tel que le Parc National de Moukalaba Doudou (PNMD), se trouvent dans les foyers historiques de la Trypanosomose Humaine Africaine (THA) (Amblard, 1996). Par ailleurs, les connaissances portant sur les vecteurs biologiques et/ou mécaniques de cette parasitose demeurent faibles sinon fragmentaires dans ces anciens foyers. En effet, de nombreux foyers historiques sont connus dans plusieurs provinces, mais ces foyers n'ont pas été prospectés depuis plus de 15 ans en raison de l'insuffisance de moyens financiers dont dispose le programme national de lutte contre la trypanosomiase humaine africaine du pays (Kohagne et al., 2011). Pourtant, des études récentes, conduites dans l'un des anciens foyers de la THA ont montré que des cas sporadiques de la THA sont diagnostiqués de manière passive par les structures sanitaires de la ville (Kohagne et al., 2011).

Un grand nombre d'agents pathogènes pour l'homme (virus, bactéries, parasites) sont transmis par des arthropodes dans ces parcs et anciens foyers de la THA (Wilcox et Ellis, 2006 ; Mavoungou, 2007). De plus, avec les changements globaux (climatiques, écologiques, démographiques et économiques) que nous observons, on assiste à une résurgence des maladies vectorielles ou encore l'émergence de nouvelles endémies (Wilcox et Ellis, 2006). Par ailleurs, avec l'arrivée importante des touristes dans ces parcs, les contacts entre populations humaines et animales seront facilités. Finalement, l'une des conséquences possibles de ces contacts sera une augmentation de la probabilité de rencontre hôtes-vecteurs et donc des échanges de pathogènes entre l'Homme, la faune et le milieu (Wilcox et Ellis, 2006). Cependant, aucune stratégie de lutte n'est efficace à long terme si les populations vectorielles cibles ne sont pas bien connues. Une meilleure connaissance des systèmes vectoriels permet de rationaliser la lutte en diminuant le rapport coût-efficacité du contrôle tout en étant attentif à l'environnement, dans la perspective de développement durable (IRD, 2011).

Bien que l'importance des glossines dans la transmission des trypanosomes soit connue (Kohagne et al., 2011), elle a longtemps masqué celle des stomoxes (Mihok, 2002 ; Desquesnes et al., 2005). Pourtant, ces derniers, qualifiés de vecteurs mécaniques ont des effets directs et indirects (transmission de pathogènes) qui peuvent avoir d'importantes conséquences médicales et même économiques dans les zones d'élevage (Mihok, 2002 ; Desquesnes et al., 2005 ; Jacquiet et al., 2014).

C'est dans ce cadre qu'une étude sur l'inventaire des stomoxes a été réalisé dans le PNMD en particulier dans deux biotopes différents: village Doussala et la forêt attenant à ce village. L'objectif de cette étude était de déterminer la densité apparente (DAP) et la diversité spécifique des stomoxes dans ces biotopes afin de connaitre leur rôle potentiel dans la transmission de la THA dans cet ancien foyer.

\section{MTERIEL ET METHODES \\ Zone d'étude}

L'étude a été menée dans le Parc National de Moukalaba-Doudou situé dans la partie sud-ouest du Gabon. Ce parc couvre une superficie de $5028 \mathrm{~km}^{2}$ (Figure 1). Il comprend plusieurs types d'habitats dans les plus importants sont : les milieux humides, les savanes, les forêts et les formations rocheuses et les grottes. Le milieu anthropisé est constitué par le village Doussala. Par ailleurs, le paysage floristique de la région comprend de nombreuses espèces qu'on ne voit pas ou 
rarement ailleurs au Gabon (Vande, 2012). La rivière Moukalaba constitue le principal affluent de la Nyanga. Elle serpente une plaine couverte d'une mosaïque de forêts et de savanes. La faune sauvage est représentée par plusieurs espèces de grands mammifères dont les buffles (Syncerus caffer nanus), les éléphants (Loxodonta africana cyclotis), les chimpanzés (Pan troglodytes), les gorilles ( $G$. gorilla gorilla) et les céphalophes (Cephalophus spp.). L'avifaune comprend plusieurs espèces dont euplecte monseigneur (Euplectes hordeaceus) (Vande, 2012).

Le climat de cette région est de type équatorial caractérisé par une saison sèche de quatre à cinq mois, de Mai à Septembre; et une saison de pluie de sept à huit mois, allant d'Octobre à ou Mai pratiquement ininterrompue (Takenoshita et al., 2008; Ebang et Juichi, 2014). La pluviométrie annuelle moyenne du parc est de $2000 \mathrm{~mm}$ de précipitations dans l'extrême nord et seulement $1600 \mathrm{~mm}$ dans le sud. Les températures varient en fonction des saisons. La différence entre les mois les plus frais et les mois les plus chauds (Mars et avril) est de l'ordre de 3 à $4{ }^{\circ} \mathrm{C}$. La différence entre la température journalière maximale et minimale augmente avec l'altitude.

\section{Capture des stomoxes}

Les stomoxes ont été capturés à l'aide des pièges Vavoua (Laveissière et Grebaut, 1990). Toutes les captures ont été réalisées entre le 17 juillet et le 22 Août 2014, c'est à dire pendant la grande saison sèche.

Au total 14 pièges ont été placés dans le PNMD dont 7 au village Doussala (milieu anthropisé) et 7 en forêt. Chaque piège était distant de l'autre d'au moins $500 \mathrm{~m}$. Ces pièges ont été laissés sur place durant toute la période de l'étude. Par ailleurs, les informations relatives à l'abondance de la végétation, à la présence des animaux sauvages et domestiques, à l'ensoleillement et à la présence humaine nous ont permis de choisir chaque site de piégeage.

Les relevés quotidiens des pièges se faisaient toujours à la même heure c'est-à-dire le matin avant 7 heures. Les insectes récoltés ont été ramenés au laboratoire pour identification et dénombrement.

\section{Identification des stomoxes}

$\mathrm{Au}$ laboratoire, les stomoxes ont été séparés des autres insectes. Ensuite, la discrimination entre les différentes espèces de stomoxes a été faite à l'aide de la clé de Zumpt (1973) et du caractère morphologique complémentaire décrit par Garros et al. (2004) pour mieux séparer $S$. calcitrans et $S$. niger.

\section{Analyse des données}

La diversité spécifique des stomoxes dans les milieux explorés a été déterminée par l'indice de diversité de Shannon, qui permet de quantifier l'hétérogénéité de la biodiversité d'un milieu. Cet indice a été calculé à l'aide de la formule suivante :

$$
\mathrm{H}^{\prime}=-\Sigma(\mathrm{Ni} / \mathrm{N}) \times \log (\mathrm{Ni} / \mathrm{N})
$$

Avec Ni le nombre d'individus d'une espèce donnée et $\mathrm{N}$ le nombre total d'individus.

L'abondance de chaque espèce de glossine capturée a été définie par la densité apparente (DAP) représentée par le nombre d'individus par piège et par jour et calculée selon la formule suivante : $D A P=\frac{\text { Nombre de mouches capturées }}{\text { Nombre de pièges } \mathrm{x} \text { nombre de jours de capture }}$ 


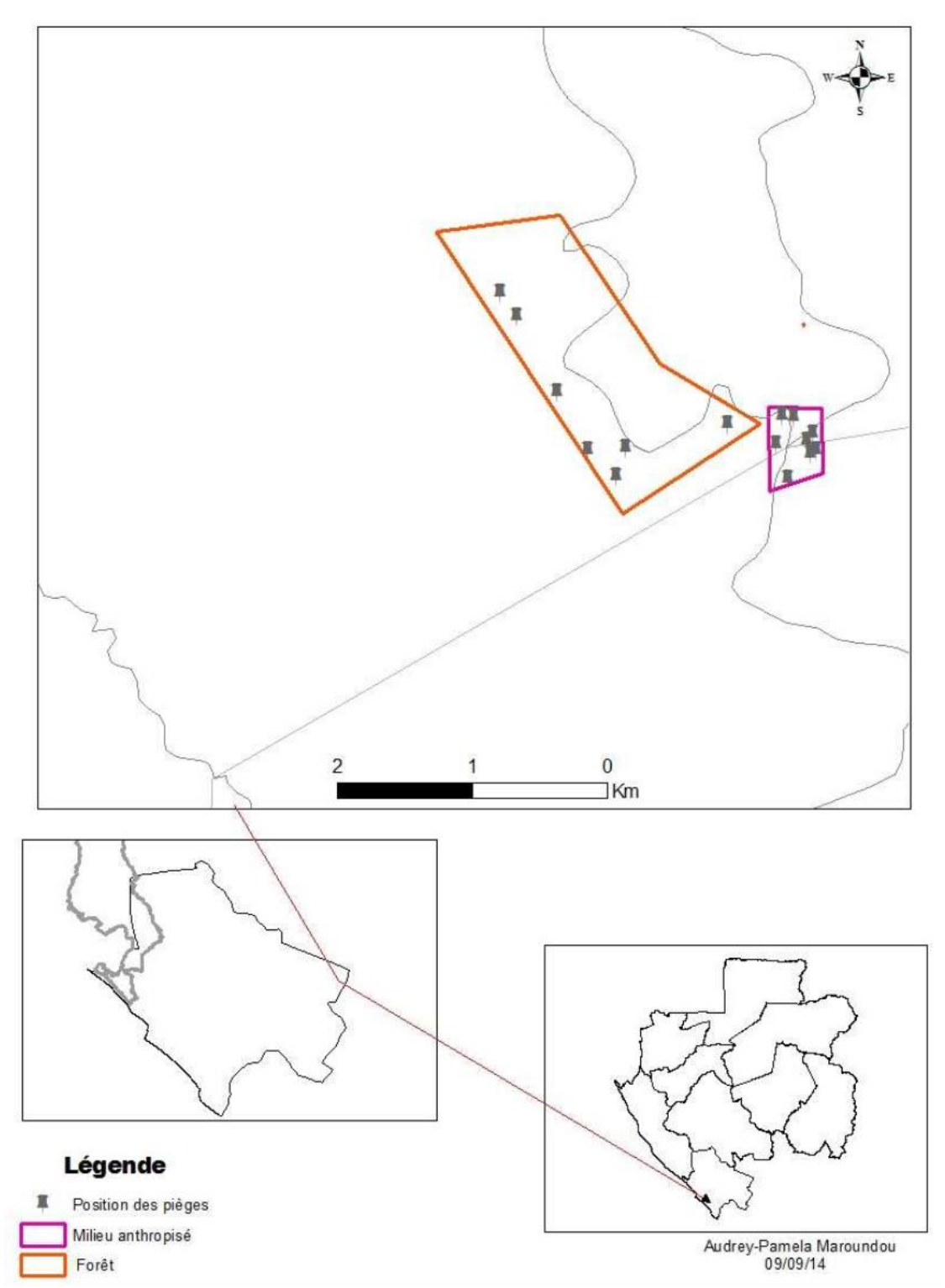

Figure 1 : Localisation du site d'étude au Gabon.

\section{RESULTATS}

$\mathrm{Au}$ total 35189 stomoxes ont été capturés en 37 jours de capture, soit une densité apparente de 67 stomoxes/piège/jour. Sur ce total, 34936 (99\%) spécimens ont été obtenus en forêt et $253(1 \%)$ au village Doussala (milieu anthropisé). Le maximum de capture a été obtenu en forêt et le minimum au village Doussala.

Sur l'ensemble des biotopes prospéctés, six (6) espèces de stomoxes ont été identifiées avec des abondances variables suivants les espèces. En effet, Stomoxys omega ( $\mathrm{DAP}=47,94)$ a été l'espèce la plus abondante, suivi de Stomoxys niger niger (DAP=19,49). Les autres espèces à savoir Stomoxys xanthomelas, Stomoxys niger bilineatus, Stomoxys calcitrans et Stomoxys transvittatus ont été très faiblement représentées avec des DAP respectives de 0,$25 ; 0,23 ; 0,012$ et 0,004 . En terme de poucentage S.omega (31\%) et S.niger niger (29\%) ont été les 
espèces les plus rencontrées. Les autres espèces ont été faiblement capturées (Figure 2).

\section{Distribution des stomoxes en fonction des biotopes échantillonnés}

$\mathrm{Au}$ village quatre espèces de stomoxes ont été identifiées avec des densités apparentes variables suivant l'espèce. En effet, $S$. niger niger $(\mathrm{DAP}=0,70)$ a été l'espèce la plus abondante, suivi de $S$. omega $(\mathrm{DAP}=$ 0,26). S. xanthomelas et S.bilineatus ont été les espèces les moins représentées avec la même densité apparente (DAP=0,001).

En forêt, une forte richesse spécifique a été observée par la présence de six (6) espèces: $S$. omega, S.niger niger, $S$. xanthomelas, S.niger bilineatus, S. calcitrans et $S$. transvittatus. $S$. omega et $S$. niger niger ont été les espèces les représentées avec des densités apparentes respectives de 95,63 et 38, 28. Les quatre autres espèces ont présenté de très faibles densités apparentes: $S$. xanthomelas (DAP $=0,49)$, S.niger bilineatus (DAP=0,46), $S$. calcitrans $(\mathrm{DAP}=0,02)$ et $S$. transvittatus $(\mathrm{DAP}=0,001)$ (Figure 3).

Toutes les espèces capturées au village ont été aussi présentes en forêt mais avec des abondances différentes. Aussi, la diversité du peuplement des stomoxes dans les deux biotopes prospectés a été évaluée par l'indice de diversité de Shannon $\left(\mathrm{H}^{\prime}\right)$. Cet indice a été de 0,87 en forêt et 0,43 en milieu anthropisé.

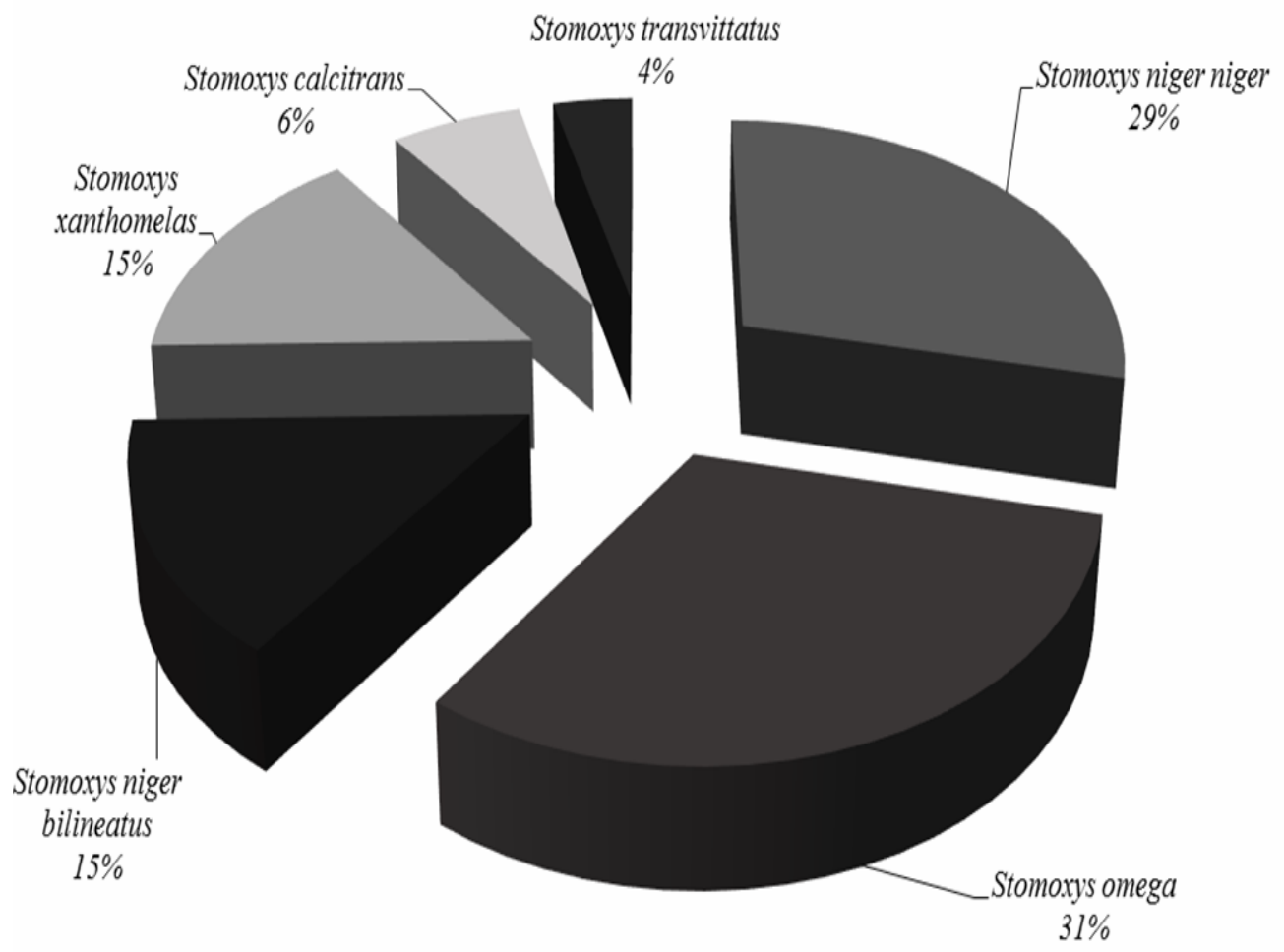

Figure 2 : Répartition spécifique des espèces de stomoxes capturés. 


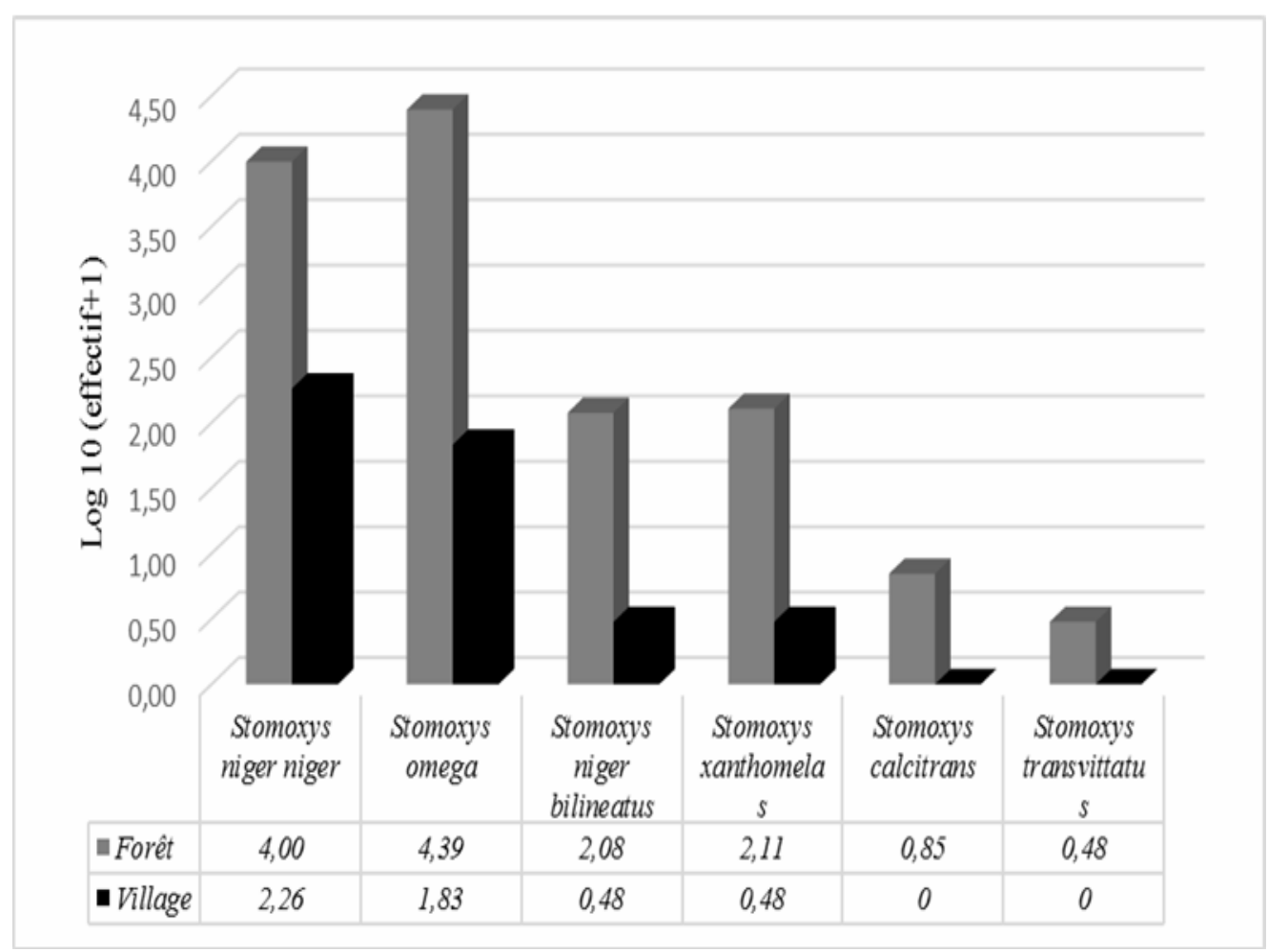

Figure 3 : Distribution spécifique des espèces de stomoxes capturées en fonction du biotope.

\section{DISCUSSION}

Les résultats de cette étude constituent des données préliminaires relatives aux stomoxes capturés dans le parc national de Moukalaba Doudou en particulier dans deux biotopes différents. Ces biotopes sont représentés par la forêt perturbée et le village Doussala attenant à cette forêt. Ces insectes sont réputés être les vecteurs mécaniques des trypanosomes, ils sont aussi vecteurs potentiels de plusieurs agents pathogènes pour l'Homme et pour les animaux (Mramba et al., 2007).

L'enquête a été realisée à l'aide d'un seul type de piège pendant une période rélativement courte à une saison unique (grande saison sèche), de ce fait ces résultats d'inventaire ne peuvent être complète donc exhaustif. Par ailleurs, seulement 14 points de captures ont été explorés. Cela n'exclut donc pas la présence d'autres espèces présentes par exemple en saison des pluies. De plus, des études conduites par Mavoungou (2007, 2008) à Makokou dans le parc National de l'Ivindo durant trois ans ont pu montrer la présence de sept (7) espèces de stomoxes qui cohabitent. Aussi, des études précédentes ont montré que l'association de plusieurs types de pièges et l'ajout d'un olfactif, l'octénol ou le dioxyde de carbone $\left(\mathrm{CO}_{2}\right)$, augmenteraient considérablement les captures des mouches hématophages (Krčmar, 2005 ; Krčmar et al. 2006; Krčmar, 2007). Ainsi, des captures longitudinales avec plusieurs types de pièges et des attractifs dans un plus grand nombre de sites sont nécessaires pour avoir une liste complète des différentes espèces de stomoxes présentes dans le parc national de Moukalaba Doudou.

En forêt six espèces (6) ont été identifiées contre quatre en milieu anthropisé, aussi le maximum de spécimens a été observé en forêt et le minimum au village. Ces différences pourraient s'expliquer par les facteurs environnementaux tels que le degré d'anthropisation $\mathrm{du}$ milieu, le faciès botanique, la présence de la faune sauvage et domestique, et la nature de la couverture 
végétale du sol. En effet, la forêt primaire est plus riche en espèces de diptères hématophages que le milieu anthropisé qui lui est adjacent. Cette diversité d'espèces serait en relation avec la stabilité du milieu forestier (omniprésence de l'humus, température plus ou moins constante, etc.), l'abondance de la grande faune, hôtes nourriciers des insectes hématophages (Broce et al., 2005); et la présence des gîtes larvaires potentiels des stomoxes (litière en forêt, crottes d'éléphants, crottes de chimpanzé, herbe en décomposition, etc.) (Mavoungou, 2007). Ces résultats corroborent ceux obtenus par Mavoungou et al. (2008) et Zinga et al. (2014a, 2014b) qui ont montré que les stomoxes sont plus abondants en forêt qu'en milieu anthropisé. De plus, le microclimat pourrait jouer aussi un rôle dans la répartition et l'abondance des différentes espèces de stomoxes.

Le substrat idéal pour assurer la ponte et le développement larvaire chez les stomoxes est un mélange de boue, de déjections (fèces, crotte et urine) et de végétaux en décomposition (Mavoungou et al. 2007, 2008 ; Jacquiet et al., 2014). Aussi, dans les conditions optimales de température, entre 25 et $35^{\circ} \mathrm{C}$ selon les espèces, les œufs éclosent en 24 heures et la durée totale du cycle est comprise entre 15 jours et 1 mois (Cauquil, 2011). Ces conditions semblent se retrouver dans la forêt et pourraient expliquer l'abondance de $S$. omega et de S.niger niger. Ces résultats sont similaires à ceux obtenus par Mavoungou (2007), qui ont montrés que S.niger niger est abondant dans les zones où la présence humaine est manifeste. En effet, la forêt attenant au village Doussala, constitue un milieu de chasse et de plantation pour les populations autochtones. De plus, par le passé cette forêt a été largement exploitée par une entreprise forestière denommée la compagnie équatoriale des bois (CEB) (Takenoshita et al., 2008; Ebang et Juichi, 2014). Quant à $S$. omega, elle demeure une espèce ubiquiste. Ainsi, les modifications apportées par l'Homme à cette forêt semblent être favorables au développement de ces deux espèces. Nos résutats corroborent également ceux obtenus par Rodriguez et al. (2005) qui ont indiqué que la perturbation des milieux favorise la croissance rapide des populations des stomoxes.

Les faibles abondances des autres espèces en forêt pourraient être liées à leurs exigences écologiques. En effet, S. niger bilineatus se retrouve beaucoup plus en zone de savane, alors que $S$ xanthomelas est inféodé à la canopée dans les zones forestières (Mavougou, 2008). Quant a $S$ calcitrans, il est inféodé aux activités humaines liées aux élevages (Jacquiet et al., 2014). S transvittatus étant une espèce ubiquiste mais les modifications apportées à cette forêt ne semblent pas être favorables à son développement.

En milieu anthropisé, on a noté de très faibles abondances et une richesse spécifique moindre. Cette faible abondance et faible richesse spécifique pourraient s'expliquer par la différenciation du paysage, à la structure du milieu pouvant engendrer des micro habitats particuliers plus ou moins favorables au développement des espèces de stomoxes (Cauquil, 2011 ; Jacquiet et al., 2014).

\section{Conclusion}

L'importance des glossines dans la transmission des trypanosomes a longtemps masqué celle des stomoxes. Pourtant, ces derniers, qualifiés de vecteurs mécaniques ont des effets directs et indirects (transmission de pathogènes) qui peuvent avoir d'importantes conséquences médicales et même économiques dans les zone d'élevage (Epstein et al., 1993). Connaître les densités apparentes et la composition spécifique des mouches hématophages dans les anciens foyers de la THA (Amblard, 1996) et parcs nationaux constituent un élément important dans la lutte anti vectorielle. Par ailleurs, la meilleure valorisation des parcs nationaux au Gabon en termes d'écotourisme passe nécessairement par la connaissance de la distribution et l'abondance des différentes espèces d'insectes vecteurs de pathogènes (Rodhain et Perez, 1985). 
Le travail sur les stomoxes du PNMD, s'inscrit dans l'étude des insectes vecteurs de pathogènes dans les parcs nationaux et dans les anciens foyers de la THA. Aussi, la problématique des arthropodes hématophages est devenue d'actualité dans le cadre de l'impact des changements globaux sur les sociétés humaines en particulier des maladies émergentes. Au total six (6) espèces de stomoxes qui vivent en sympatrie ont été identifiées dans le PNMD. L'abondance et la diversité spécifique de ces espèces varient suivant le biotope. De plus, la forte abondance des stomoxes dans ce parc nous interpelle à considérer ces insectes dans la lutte contre la THA dans cet ancien foyer. Cependant, ce résultat d'inventaire ne peut être exhaustif, car un seul type de piège a été utilisé pendant une période relativement courte (Djiteye, 1992; Amsler \& Filledier 1994; Krčmar, 2005 ; Krčmar et al. 2006; Krčmar, 2007). Par conséquent, une étude plus approfondie de la distribution spatio-temporelle de ces insectes doit être entreprise pour connaître leur dynamique saisonnière et leur impact potentiel dans la transmission de la THA dans cet ancien foyer.

\section{REMERCIEMENTS}

Ce travail a été réalisé grâce à l'appui institutionnel, financier et logistique de l'Institut de Recherche en Ecologie Tropicale (IRET-CENAREST), l'Université des Sciences et Techniques de MASUKU (USTM), le Laboratoire d'Ecologie Vectorielle (LEV-IRET), le Projet pour la Conservation De La Biodiversité En forêt Tropicale A Travers La Coexistence Durable Entre L' Homme Et l'Animal (PROCOBAH), l'Ecole Régionale Post-Universitaire d'Aménagement et de Gestion Intégrés des Forêts et Territoires Tropicaux (ERAIFTUNIKIN) et la CEEAC/PACEBO.

Les auteurs remercient $\mathrm{Mr}$ LOGNO NZABA Roye pour son aide technique très précieuse sur le terrain et Madame MAROUNDOU Audrey Pamela pour la réalisation de la carte.
Nos sincères remerciements sont également dirigés à l'adresse de Mr. Ephrem NZENGUE, Mr. Clency MIKALA, Mr. Karl HENGA BOTSIKA BOBE, Mr. Nathaniel KOUMBA, Mlle BELL Rayna Camille et Mr. Patrick ONDO OKAMA dont les remarques, suggestions et corrections ont permis d'améliorer ce manuscrit.

\section{REFERENCES}

Akoma MR. 2014. Mammifères de la forêt guinéo-Congolaise: Partage. Edition du CENAREST. 267p.

Amsler S, Filledier J. 1994. Attractivité pour les Tabanidae de l'association métacrésol/octénol : Résultats obtenus au Burkina Faso. Revue Elevage et de Médecine vétérinaire des Pays Tropicaux, 47 : 93-96.

Amblard J. 1996. Situation de la trypanosomiase humaine africaine au Gabon. Etude sur la période 19931995. Rapport interne du service des grandes endémies, Ministère de la santé, Gabon, p.18.

Bergonzini JC, Lanly JP. 2000. Les forêts tropicales. Cirad-Montpellier. p.164.

Broce AB, Hogsette J, Paisley S. 2005. Winter feeding sites of hay in round bales as major devepmental sites of Stomoxys calcitrans (Diptera: Muscidae) in pastures in spring and summer. Journal of Economic Entomology, 98: 2307-2312. DOI : https://doi.org/10.1603/0022-049398.6.2307

Cauquil L. 2011. Mise en place d'un élevage de Stomoxys calcitrans et de Stomoxys indicus et étude des sites de développement larvaire des Stomoxys indicus en Thaïlande. Thèse de doctorat, Université de Toulouse, p. 80.

Desquesnes M, Dia ML, Acapovi GL, Yoni W, Foil L, Pin R. 2005. Les Vecteurs Mécaniques des Trypanosomoses Animales : Généralités, morphologie, Biologie, Impacts et Contrôle. Identification des Espèces les plus Abondantes en Afrique de l'Ouest. Editions Cirdes, Imprimerie de l'Avenir : Burkina Faso, p.68 
Djiteye A. 1992. Aperçu sur l'efficacité comparative de différents pièges et odeurs contre les mouches piqueuses (Diptera : Tabanidae et Muscidae) d'importance vétérinaire. In Premier séminaire International sur les Trypanosomoses Animales non Transmises par les Glossines, Annecy (France), 14-16 octobre 1992.

Ebang EGW, Juichi Y. 2014. Use of tool sets by chimpanzees for multiple purposes in Moukalaba- Doudou National Park, Gabon. Primates, p.6. DOI: 10.1007/s10329-014-0431-5.

Epstein PR, Ford TE, Colwell RR. 1993. Marine ecosystems: emerging diseases as indicators of change. Lancet, 342: 12171219.

FAO. 2002. Global forest resources assessment 2000 Main report. FAO, forestry paper $n^{\circ} 140$. Eds. Lavoisier.

Garros C, Gilles J, Duvallet G. 2004. Un nouveau caractère morphologique pour distinguer Stomoxys calcitrans et $S$. niger (Diptera : Muscidae). Comparaison de populations de l'île de La Réunion. Parasite, 11: 329-332.

IRD (Institute de Recherche pour le Développement). 2011. L'IRD en Afrique centrale: Cameroun - Centrafrique Congo - Gabon - Guinée équatoriale R.D. Congo. IRD/DIC, Mai 2011. p.62.

Jacquiet P, Rouet D, Bouhsira, Salem A, Liénard E, Franc M. 2014. Population dynamics of Stomoxys calcitrans (L.) (Diptera: Muscidae) in southwestern France. Revue de Médecine Vétérinaire. 165(9-10) : 267271.

Kohagne TL, Gounoue KR, Mengue MP, Kaba D, Louis FJ, Mimpfoundi R. 2011. Enquête entomologique dans le foyer historique de trypanosomose Humaine Africaine de Bendjé (Gabon). Parasite, 18(4): 303-309.

DOI : [10.1051/parasite/2011184303]

Krčmar S. 2005. Reponse of horse flies (Diptera, Tabanidae) to different olfactory attractants. Biologia, 60: 611613.
Krčmar S, Mikuska A, Merdic E. 2006. Response of Tabanidae (Diptera) to different naturel attractants. Journal of Vector Ecology. 31: 262-265. DOI: https://doi.org/10.3376/10811710(2006)31[262:ROTDTD]2.0.CO;2

Krčmar S. 2007. Reponse of horse flies (Diptera, Tabanidae) to canopy traps baited with 4-methylphenol, 3isopropylphenol, and naphthalene. Journal of Vector Ecology. 32: 188192.

Mavoungou JF. 2008. Ecologie et rôle vecteur des stomoxes (Diptera : Muscidae) au Gabon. Thèse de doctorat. Université Montpellier III, p.137.

Mavoungou JF, Jay-robert P, Gilles J, Atsame Edda A, Duvallet G. 2008. Ecologie des stomoxes (Diptera: Muscidae) au Gabon. I. Premier inventaire dans différentes zones écologiques. Parasite, 15: 27-34. DOI: http://dx.doi.org/10.1051/parasite/20081 51027

Mihok S. 2002. The development of multipurpose trap (the Nzi) for tsetse and other biting flies. Bull. Entomol. Res. 92: 385-403. DOI:10.1079/BER2002186

Mramba F, Broce $\mathrm{AB}$, Zurek L. vector competence of stable flies, Stomoxys calcitrans L. (Diptera: Muscidae), for Enterobacter sakazakii. Journal of vector Ecology. 32 (1): 134-139. DOI : https://doi.org/10.3376/10811710(2007)32[134:VCOSFS]2.0.CO;2

PFBC (Partenariat pour les Forêts du Bassin du Congo). 2006. Les forêts du Bassin du Congo: Etat des Forêts 2006. PFBC, $256 \mathrm{p}$

Rodhain F, Perez C. 1985. Les Muscidae Stomoxyinae. In : Précis d'Entomologie Médicale et Vétérinaire. Paris, France, Maloine, p 394-395.

Rodriguez-Batista Z, Leite RC, Oliveira PR, Lopes CML, Borges LMF. 2005. Populational dynamics of Stomoxys calcitrans (Linnaeus) (Diptera : Muscidae) in three biocenosis, Minas Gerais, Brazil. Veterinary Parasitology. 130: 343-346.

Takenoshita Y, Ando C, Iwata Y, Yamagiwa J. 2008. Fruit phenology of the great habitat in the Moukalaba-Doudou 
National Park, Gabon. African Study Monograph Supplementary.39: 23-39.

UICN. 1996. Guide de la Convention sur la Diversité Biologique. 193 p.

Vande Weghe JP. 2006. Ivindo et Mwagna. Eaux noires, forêts vierges et baïs. Wildlife Conservation Society, Libreville, Gabon: p.272.

Vande W.J.P. 2012. MOUKALABA DOUDOU. Agence National des Parcs Nationaux (ANPN), Libreville, Gabon. 296 p.

Wilcox BA, Ellis B. 2006. Les forêts et les maladies émergentes chez l'homme. Unasylva. 57 : 11-18.

Zinga Koumba CR, Mbang Nguema OA, Kohagne TL, Acapovi-Yao GL, Obame OKP, Mutambwe S, Mavoungou JF. 2014a. Contribution à l'évaluation de la diversité des vecteurs biologiques de la Trypanosomose Humaine Africaine et de leur activité journalière dans le Parc National de l'Ivindo (Nord-est Gabon). Journal of Applied Biosciences, 80:7060-7070. DOI: http://dx.doi.org/10.4314/jab.v80i1.8

Zinga koumba CR, Mbang Nguema OA, Mavoungou JF, Obame Ondo KP. 2014b. Ecodistribution des tabanidés, glossines et stomoxes le long d'un transect forêt Primaire-village au Gabon. International Journal of Biological and Chemical Sciences. 8 (1): 167-181. DOI: http://dx.doi.org/10.4314/ijbcs.v8i1.16

Zumpt F. 1973. Diptera: Muscidae; Taxonomy and biology of species. In The Stomoxyinae Biting flies of the World. Gustav Fischer Verlag: Stuttgart, Germany, 20-97. 